\title{
Laterally spreading features of gastrointestinal stromal tumors: A clinicopathological study
}

\author{
SUSUMU MATSUKUMA ${ }^{1,2}$, MICHINORI MURAYAMA ${ }^{3}$, YOSHITAKA UTSUMI $^{1}$, KOJI SUMI $^{1}$, \\ KOSUKE MIYAI $^{1}$ and HIROAKI TAKEO ${ }^{1}$ \\ ${ }^{1}$ Department of Pathology, ${ }^{2}$ Health Care Center and ${ }^{3}$ Division of Surgery, Japan Self-Defense Forces Central Hospital, \\ Setagaya-ku, 154-8532 Tokyo, Japan
}

Received November 4, 2017; Accepted April 4, 2018

DOI: $10.3892 /$ or.2018.6360

\begin{abstract}
To elucidate the histopathological features of laterally spreading gastrointestinal stromal tumors (GISTs), we retrospectively examined 52 GISTs grossly completely resected from 50 patients. Laterally spreading features were identified in 7 GISTs (13\%), and were localized within non-thickened regions of the muscularis propria adjacent to the main GISTs, ranging in length from 0.12 to $0.7 \mathrm{~cm}$ (mean, $0.3 \mathrm{~cm}$ ). The laterally spreading features involved the muscular surgical margins in 2 cases. The morphologies of the laterally spreading cells resembled those of tumor cells in 4 cases, but were comprised of more slender spindle cells with smaller nuclei compared with those in the respective main GISTs. Compared with the main GISTs, $\mathrm{KIT}^{+}$and discovered on GIST $1^{+}$immunostaining features of the spreading lesions were similar in 4 cases, and were weaker or diminished in the other 3 cases. There were no differences in $\mathrm{CD} 34^{+}$staining features between the main GISTs and the laterally spreading lesions. One patient with laterally spreading GIST succumbed to the disease 2.5 years after the surgery, while the other 6 patients were alive without the recurrence of disease 0.4-19.2 years after the surgery. The laterally spreading features were associated with a pedunculated GIST $(\mathrm{P}=0.006)$, but not older age $(\mathrm{P}=0.312)$, sex $(\mathrm{P}=0.969)$, tumor size $(\mathrm{P}=0.430)$, mucosal invasion $(\mathrm{P}=0.666)$ or higher risk category $(\mathrm{P}=0.872)$. Results of the present study indicate that resection of a $\geq 1-\mathrm{cm}$ muscular safety margin, and not mucosa or submucosa, is required for
\end{abstract}

Correspondence to: $\mathrm{Dr}$ Susumu Matsukuma, Department of Pathology, Japan Self-Defense Forces Central Hospital, 1-2-24 Ikejiri, Setagaya-ku, Tokyo 154-8532, Japan

E-mail: skuma@cocoa.plala.or.jp

Abbreviations: DOG1, discovered on GIST 1; GI, gastrointestinal; GIST, gastrointestinal stromal tumor; ICCs, interstitial cells of Cajal; S-100, S-100 protein; $\alpha$-SMA, $\alpha$-smooth muscle actin; UICC, Union for International Cancer Control

Key words: gastrointestinal stromal tumor laterally spreading, surgical margin, $\mathrm{R} 1$ resection microscopically negative surgical margins, particularly for pedunculated GISTs.

\section{Introduction}

Gastrointestinal stromal tumors (GISTs) are the most common mesenchymal tumors of the gastrointestinal (GI) tract, and arise from interstitial cells of Cajal (ICCs) or their precursor cells (1-5). GISTs tend to form well-circumscribed, protruding nodules without diffuse infiltration, so that local resection is often initially an adequate therapy (3,6-8). A 1-2 cm surgical safety margin is thought to be required for grossly and microscopically complete resection of GISTs (8-10), referred to as an $\mathrm{R} 0$ resection in the Union for International Cancer Control (UICC) system (11). Novitsky et al (9) demonstrated that all 50 surgically removed GISTs with a grossly $1-2 \mathrm{~cm}$ margin beyond the tumors exhibited microscopic negative margins ranging from 0.2 to $4.5 \mathrm{~cm}$. Based on this, Everett et al (10) deduced a possible $0.5-\mathrm{cm}$ length microscopic extension of GISTs. To the best of our knowledge, however, detailed histopathological examination of such laterally extending or spreading lesions of GISTs has not been previously conducted. Therefore, we examined the incidence and the histopathological features of these laterally spreading lesions of GISTs in the present study.

\section{Materials and methods}

Patients and GISTs. We examined a total of 52 GISTs grossly completely removed from 50 patients, which were retrieved from the surgical pathology files (1994-2016, October) of the Department of Pathology, Japan Self-Defense Forces Central Hospital, and the surgical pathology files (1996-2017, April) of the Division of Pathology, Mishuku Hospital, Tokyo, Japan. All GISTs were confirmed to be immunohistochemically positive for KIT (1:100; polyclonal; cat. no. A4502; Dako; Agilent Technologies, Inc., Santa Clara, CA, USA) and CD34 (1:100; NU-4A1; cat. no. 413361; Nichirei Biosciences, Inc., Tokyo, Japan). Clinical findings were obtained from medical charts and request forms for surgical pathology examination. Patients consisted of 39 men and 11 women, and ranged in age from 33 to 88 years (mean, 63.9 years). GISTs were located on the esophagus (2), stomach (38), small intestine (11 in total: 
Table I. Clinicopathological findings of 52 GISTs removed from 50 patients.

Patients [n=50 patients]

Age (years)

Sex, male/female, $\mathrm{n}$

GIST location

Esophagus/stomach/small intestine/large intestine, n (\%)

Tumor size $(\mathrm{cm})$

Macroscopic type

Exophytic/pedunculated $\mathrm{b} /$ dumbbell-shaped, n (\%)

Microscopic findings

Ulceration, $\mathrm{n}(\%)$

Spindle-cell/epithelioid-cell predominant, $\mathrm{n}(\%)$

High mitotic rate (>5 per $50 \mathrm{HPFs}), \mathrm{n}(\%)$

Mucosal invasion, $\mathrm{n}(\%)$

Skeinoid fibers, $\mathrm{n}(\%)$

Laterally spreading features, $\mathrm{n}(\%)$

$\mathrm{R} 1$ resection $^{\mathrm{c}}, \mathrm{n}(\%)$

Immunohistochemical positivity

KIT/CD34/DOG1/focal SMA/focal S-100 protein, n (\%)

Risk category of GIST ${ }^{\circ}$

Very low/low/intermediate/high, n (\%)
33-88 (mean, 63.9)

$39 / 11$

$2(4 \%) / 38(73 \%) / 11(21 \%) / 1(2 \%)$

0.09-20 (mean, 4.56)

$29(56 \%) / 8(15 \%) / 8(15 \%)$

$13(25 \%)$

$43(83 \%) / 9(17 \%)$

$13(25 \%)$

$14(27 \%)$

$8(15 \%)$

$7(13 \%)$

$2(4 \%)$

$52(100 \%) / 52(100 \%) / 37(97 \%)^{\mathrm{d} / 14}(27 \%) / 4(8 \%)$

$15(29 \%) / 16(31 \%) / 11(21 \%) / 10(19 \%)$

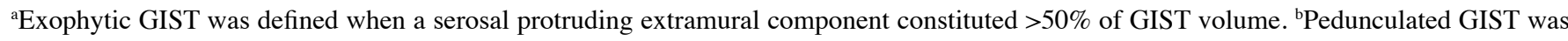
defined when an exophytic GIST had a relatively narrow pedicle. ${ }^{c}$ Macroscopically complete resection with microscopically positive surgical

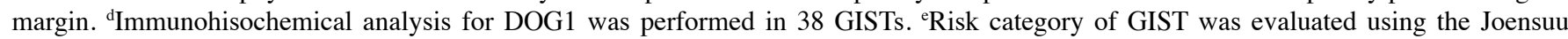
criteria (12). DOG1, discovered on gastrointestinal tumor 1; GIST, gastrointestinal stromal tumor; HPFs, high power fields; SMA, $\alpha$-smooth muscle actin.

Duodenum, 2; jejunum, 2; ileum, 1; and not otherwise specified, 6) and cecum (1). Sixteen minute or small GISTs were incidentally detected in other diseases. The present study was a retrospective study, which was performed according to the Declaration of Helsinki, and was approved by the Medical Research Ethics Committees of the Japan Self-Defense Forces Central Hospital (approval no. 28-014) and Mishuku Hospital (approval no. 2016-04).

GIST examination. In the present study, we defined 'exophytic' GISTs, as those with serosal protruding extramural components constituting $>50 \%$ of the tumor volume. 'Dumbbell' -shaped GISTs were also found (2), but were re-classified in this study as either exophytic or non-exophytic, according to the aforementioned definition. Exophytic GISTs attached to the GI wall with a relatively narrow pedicle were called 'pedunculated' GISTs. When a laterally spreading lesion was present, we calculated its length from the outline of the main GIST or from the pedicle of the pedunculated GIST. Histology of GISTs was divided into 2 types, that is, spindle- or epithelioid-cell predominant. The risk category of each GIST was evaluated using the Joensuu criteria (12). All 10-20\% buffered formalin-fixed and paraffin-embedded representative specimens were available. Select serial $4 \mu \mathrm{m}$-thick sections were re-cut and immunostained for discovered on GIST 1 (DOG1) (1:100; clone no. K9; cat. no. NCL-L-DOG-1; Leica Biosystems, Newcastle, UK), $\alpha$-smooth muscle action (SMA) (clone no. 1A4; cat. no. 412021; Nichirei Biosciences, Inc., Tokyo, Japan; prediluted) and
S-100 protein (S-100) (polyclonal; cat. no. 422091; Nichirei Biosciences, Inc.; prediluted).

Statistical analysis. Associations between the laterally spreading features and other clinicopathological findings were analyzed using the Chi-square test with the Yates' correlation and the unpaired t-test. Significance was set at $\mathrm{P}<0.05$.

\section{Results}

Table I summarizes the clinicopathological findings of the 52 GISTs. Of the 52 GISTs, 29 (56\%) were exophytic, $8(15 \%)$ were pedunculated, $8(15 \%)$ were dumb-bell shaped, $13(25 \%)$ exhibited ulceration, 43 (83\%)/9 (17\%) were spindle-cell/epithelioid-cell predominant, 13 (25\%) had a high mitotic rate (>5/50 high-power fields), 14 (27\%) exhibited mucosal invasion, and $8(15 \%)$ contained skeinoid fibers. Focal SMA and S-100 positivity was found in $14(27 \%)$ and $4(8 \%)$ GISTs, respectively. In 38 GISTs immunostained for DOG1, $37(97 \%)$ exhibited positivity. Eleven (21\%) and 10 GISTs (19\%) were assessed as intermediate and high risk, respectively. Laterally spreading features were identified in 7 GISTs (13\%). In 2 GISTs, the laterally spreading tumor cells involved the surgical margin, and were referred to as an $\mathrm{R} 1$ resection according to the UICC system (11). No other R1 cases were found.

A summary of the clinicopathological findings of laterally spreading GISTs is provided in Table II. Of these, 1 arose in 


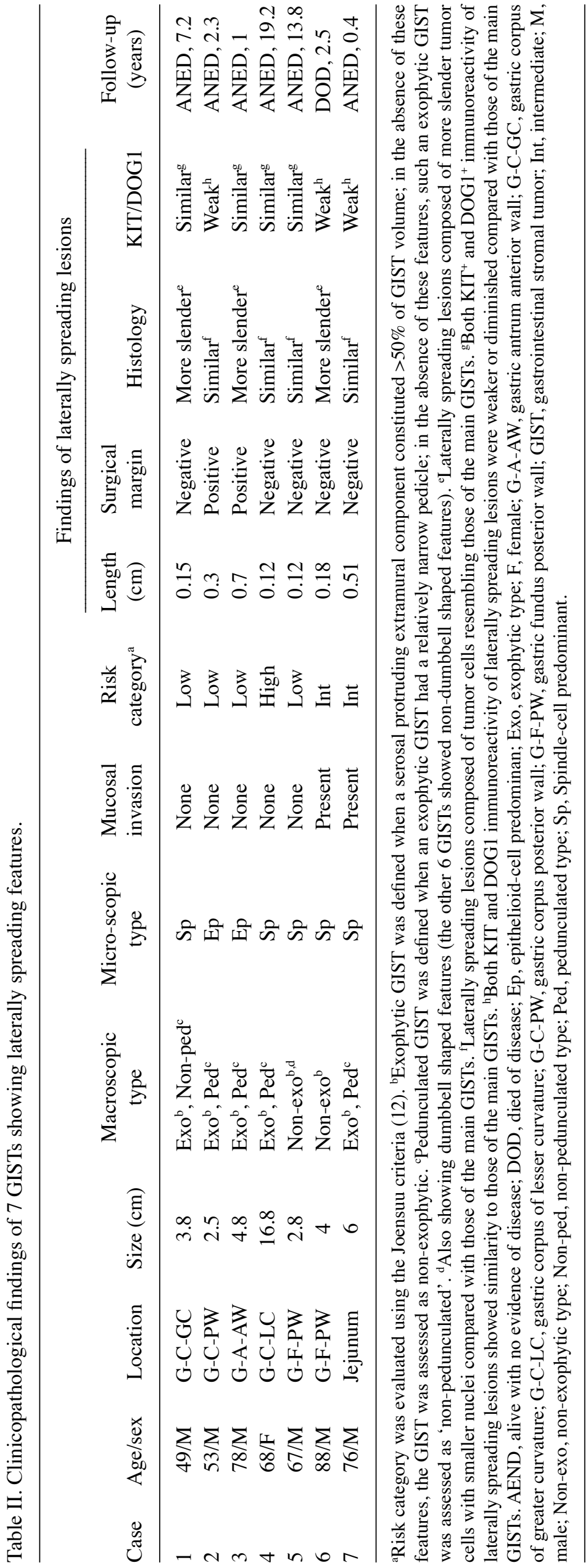



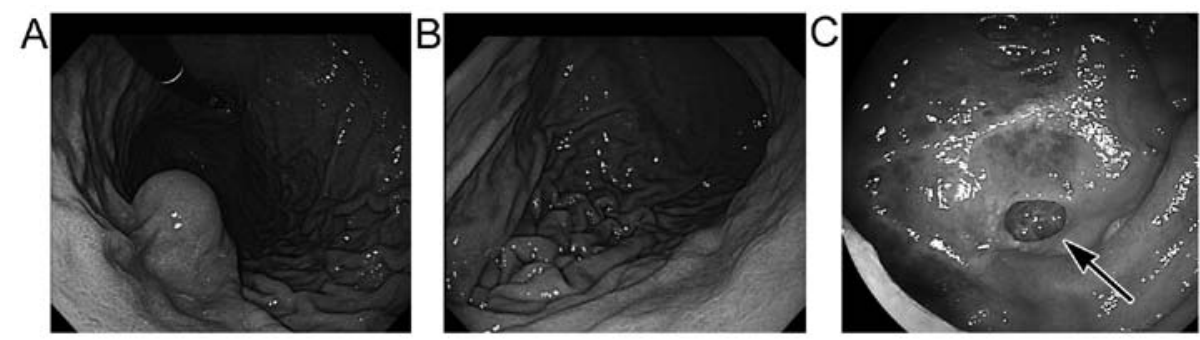

Figure 1. Endoscopic features of laterally spreading gastrointestinal stromal tumors (GISTs). (A) Protruding submucosal lesions of GIST at the greater curvature of the gastric corpus (case 1). (B and C) No elevated lesions were observed in the posterior wall of the gastric corpus (B, case 2) and jejunum (C, case 7) attached to the exophytic GISTs, other than focal mucosal depressed lesions (C, arrow). Compare with macroscopic features shown in Figs. 2 and 3.

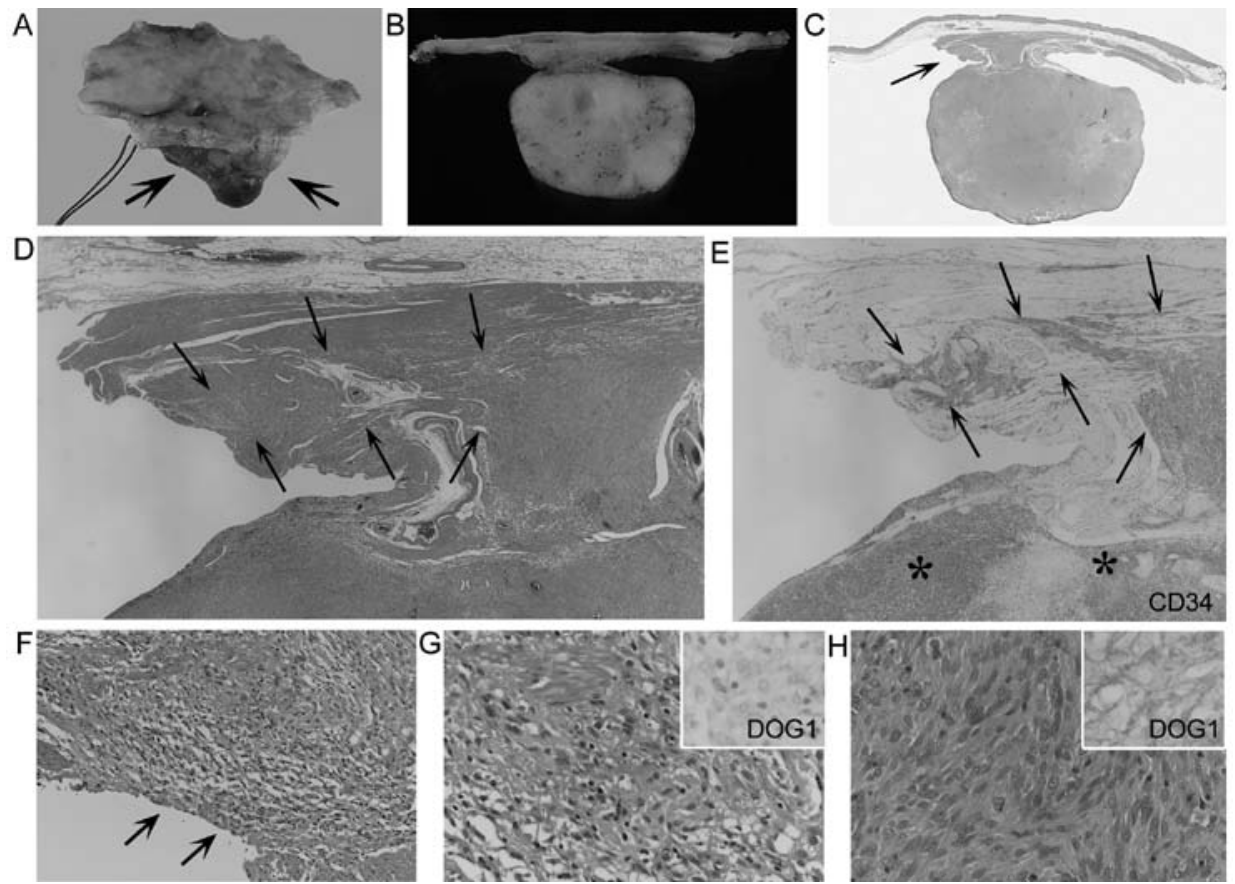

Figure 2. Laterally spreading gastrointestinal stromal tumor (GIST) (case 2). (A and B) An exophytic GIST (A, arrows) and a cut section (B) revealing a pedunculated shape. (C) H\&E-stained loupe view showing widely removed mucosal and submucosal margins with limited non-thickened muscularis propria (arrow). (D and E) Low-power view of H\&E-stained intramuscular spreading lesions (D, arrows), and their CD34+ features (E, arrows), resembling those of the main GIST (E, asterisks) (magnification, x20 for both). (F) H\&E-stained moderate-power view of intramuscular spreading lesions, which involve the surgical margin (arrows) (magnification, x200). (G and H) H\&E-stained high-power view of laterally spreading tumor cells (G) and tumor cells of the main GIST (H), showing some resemblance between both types of cells. Discovered on GIST 1 (DOG1) ${ }^{+}$features of laterally spreading tumor cells (G, inset) were weaker than those of the main GIST (H, inset) (magnification, x400 for all).

the jejunum, while the other 6 were present on the gastric walls, including the posterior wall of fundus (2 cases), corpus ( 3 cases) and antral anterior wall (1 case). Five (71\%) were exophytic, while 4 exhibited pedunculated features. Endoscopically, submucosal tumor-like protrusions or mildly elevated features were observed in 4 GISTs (Fig. 1A; cases 1, 3, 5 and 6) and concomitant ulceration was found in one (case 6). However, these endoscopic features did not appear to be different from those of other GISTs without laterally spreading lesions. The other 3 laterally spreading GISTs (cases 2, 4 and 7) exhibited no elevated tumorous features endoscopically (Fig. 1B and C), although one of them (case 7) had well-demarcated, depressed lesions (Fig. 1C). Histologically, the laterally spreading features were not found in the mucosa or submucosa, and were localized within the muscularis propria without significant thickening adjacent to the main GISTs (Figs. 2-4). The lengths of these spreading lesions ranged from 0.12 to $0.7 \mathrm{~cm}$ (mean, $0.3 \mathrm{~cm}$ ). In the $2 \mathrm{R} 1$ resection cases, the removed muscular layers adjacent to the main GISTs were relatively limited, and their surgical margins were involved by the spreading lesions (Fig. 2C and F). These spreading cells histologically resembled those of tumor cells of the main GISTs in 4 cases (Figs. 2G and $\mathrm{H}$, and $3 \mathrm{~B}, \mathrm{~F}$ and $\mathrm{I}$ ). In another 3 cases, the spreading cells consisted of more slender spindle cells with smaller nuclei, compared with tumor cells of the main GISTs (Fig. 4D and F). Compared with immunohistochemical features of the main GISTs, $\mathrm{KIT}^{+}$and DOG1 staining of the spreading lesions were similar in 4 cases (Fig. $4 \mathrm{E}$ and $\mathrm{G}$ ), but were weaker or diminished in the other 3 cases (Fig. 2G, inset and H, inset). There were no differences of $\mathrm{CD}^{+} 4^{+}$staining between the main GISTs and the spreading lesions (Figs. 2E and 4C). No SMA or S-100 positivity was found in the spreading spindle cells. One patient (case 6) succumbed to the disease 2.5 years 

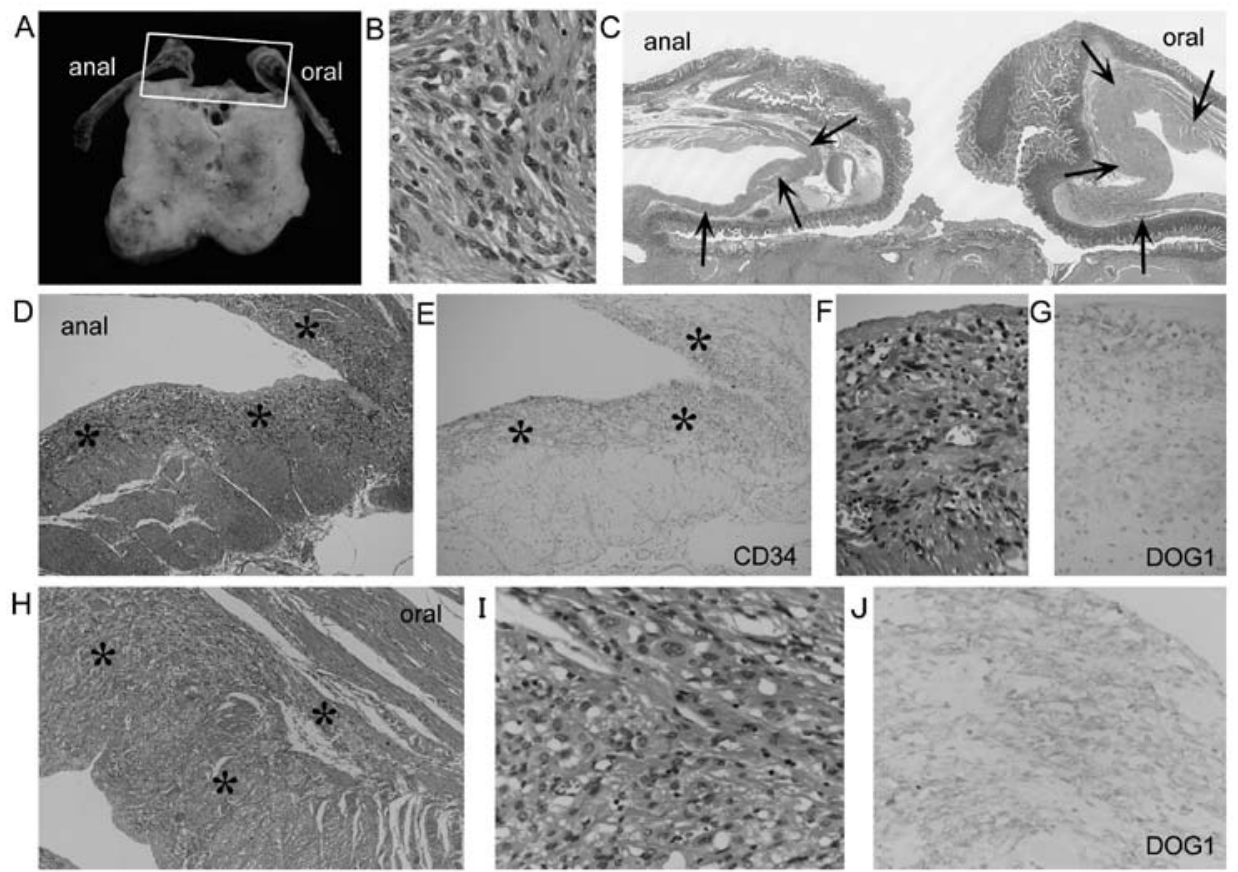

Figure 3. Laterally spreading gastrointestinal stromal tumor (GIST) (case 7). (A) Cut section of an exophytic GIST revealing a pedunculated mass. (B) High-power view of the main GIST (magnification, x400). (C) H\&E-stained loupe view of the outlined area in Fig. 2A, containing laterally spreading lesions (arrows) within both anal and oral non-thickened muscular walls. (D and E) Low-power view of H\&E-stained anal spreading lesions chiefly involving the outer layer of the muscularis propria (D, asterisks) and their CD34+ features (E, asterisks) (magnification, x100 for both). (F and G) High-power view of H\&E-stained laterally spreading tumor cells and their discovered on GIST 1 (DOG1) features (G) (magnification, x400 for both). (H) Moderate-power view of H\&E-stained oral spreading lesions (asterisks) (magnification, x100). (I and J) High-power view of H\&E-stained oral spreading tumor cells (I) and their $\mathrm{DOG1}^{+}$features (J). These DOG1 ${ }^{+}$features resembled those of the main GIST (data not shown) (magnification, $\mathrm{x} 400$ for both).
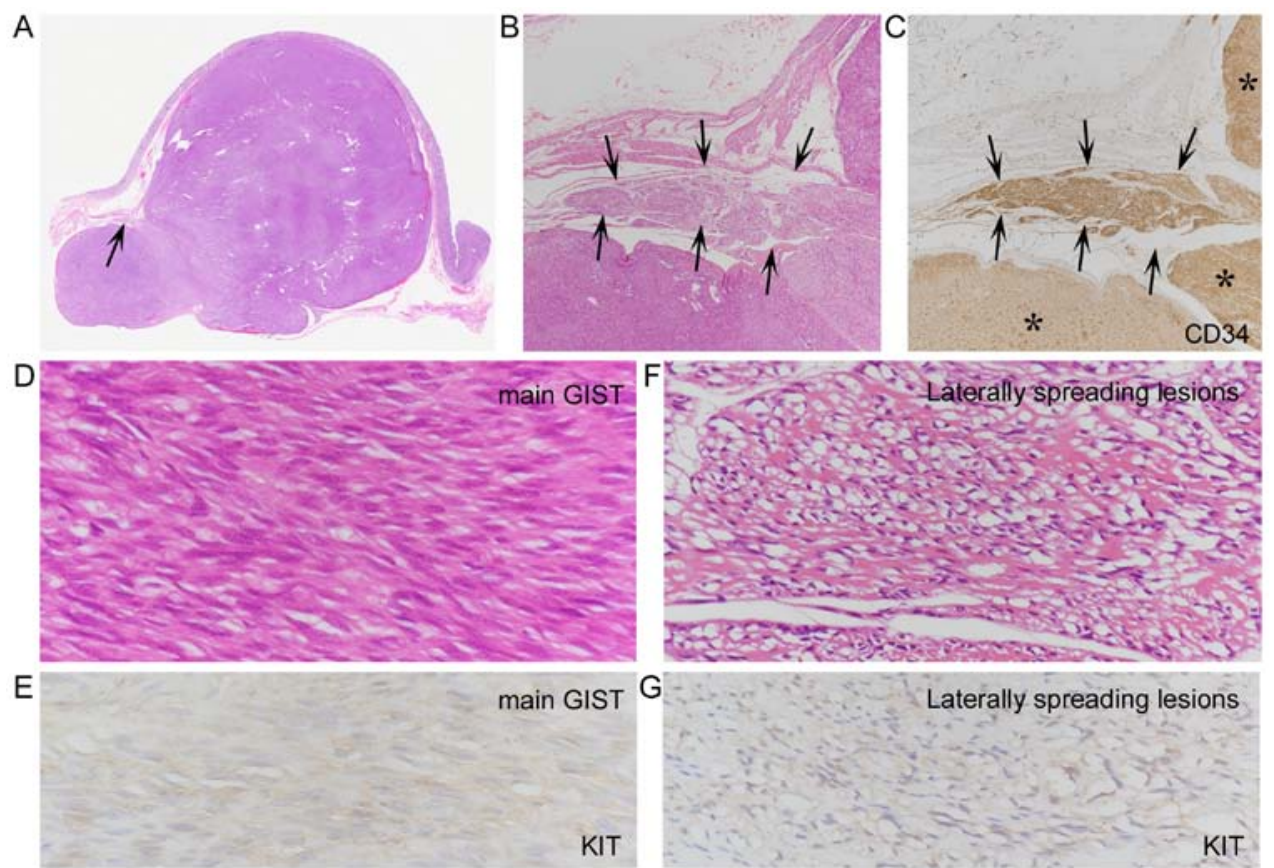

Figure 4. Laterally spreading gastrointestinal stromal tumor (GIST) (case 5). (A) Loupe view of a H\&E-stained non-exophytic GIST exhibiting minimal lateral spreading (arrow). (B and C) Low-power view of H\&E-stained intramuscular spreading lesions (B, arrows) and their CD34 $4^{+}$features (C, arrows), exhibiting features similar to those of the main GIST (C, asterisks) (magnification, $\mathrm{x} 40$ for both). (D-G) High-power views of tumor cells in the main GIST (D and E) and in laterally spreading lesions (F and G). Compared with tumor cells of the main GIST (D), hematoxylin and eosin-stained spreading tumor cells (F) were more slender with smaller nuclei. However, there were no significant differences in KIT staining features between the main GIST (E) and the spreading lesions (G) (magnification, $\mathrm{x} 400$ for D-G).

after the surgery. In this case, the autopsy revealed a $5.5-\mathrm{cm}$ recurrent growth of the GIST at the gastric excision site and multinodular peritoneal seeding in the upper abdominal cavity. The other 6 patients with laterally spreading GISTs 
Table III. Relationship between the laterally spreading GISTs and other clinicopathological variables.

Presence of laterally spreading lesions of GISTs

\begin{tabular}{|c|c|c|c|}
\hline & & & \\
\hline Variables & Yes $(n=7)$ & No $(n=45)$ & P-value \\
\hline Age, range (mean), years & $49-88$ (68.4) ( $\mathrm{n}=7$ patients) & $33-81$ (63.2) ( $\mathrm{n}=43$ patients) & 0.312 \\
\hline Sex (Male/female), n & 6/1 (n=7 patients) & $33 / 10$ ( $\mathrm{n}=43$ patients $)$ & 0.969 \\
\hline Gastric/non-gastric, $\mathrm{n}$ & $6 / 1$ & $32 / 13$ & 0.725 \\
\hline Tumor size (mean), $\mathrm{cm}$ & $2.5-16.8(5.81)$ & $0.09-20(4.37)$ & 0.430 \\
\hline Exophytic type ${ }^{a}, \mathrm{n}$ & 5 & 24 & 0.626 \\
\hline Pedunculated type ${ }^{\mathrm{b}}, \mathrm{n}$ & 4 & 4 & $0.006^{\mathrm{c}}$ \\
\hline Dumbbell-shaped features, $\mathrm{n}$ & 1 & 7 & 0.634 \\
\hline Ulceration, $\mathrm{n}$ & 2 & 11 & 0.977 \\
\hline Spindle-cell/epithelioid-cell predominant, $\mathrm{n}$ & $5 / 2$ & $38 / 7$ & 0.757 \\
\hline High mitotic rate ( $>5$ per $50 \mathrm{HPFs}$ ), $\mathrm{n}$ & 1 & 12 & 0.815 \\
\hline Mucosal invasion, $\mathrm{n}$ & 2 & 13 & 0.666 \\
\hline Skeinoid fibers, $\mathrm{n}$ & 1 & 7 & 0.634 \\
\hline Risk category ${ }^{\mathrm{c}}$, intermediate risk + high risk, $\mathrm{n}$ & 3 & 17 & 0.872 \\
\hline
\end{tabular}

${ }^{a}$ Exophytic GIST was defined when a serosal protruding extramural component constituted $>50 \%$ of the GIST volume. ${ }^{b}$ Pedunculated GIST

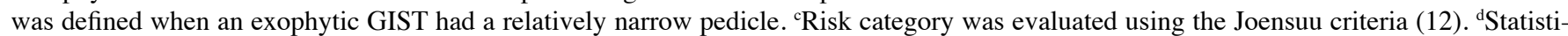
cally significant. GIST, gastrointestinal stromal tumor; HPFs, high-power fields.

were alive 0.4-19.2 years after the surgery, with no evidence of disease.

The laterally spreading features were significantly associated with pedunculated GISTs $(\mathrm{P}=0.006)$, but not with older age $(\mathrm{P}=0.312)$, sex $(\mathrm{P}=0.969)$, tumor location (gastric or non-gastric) $(\mathrm{P}=0.725)$, tumor size $(\mathrm{P}=0.430)$, exophytic type $(\mathrm{P}=0.626)$, dumb-bell shape $(\mathrm{P}=0.634)$, ulceration $(\mathrm{P}=0.977)$, microscopic type (spindle- or epithelioid-cell predominant $(\mathrm{P}=0.757)$, high mitotic rate $(\mathrm{P}=0.815)$, mucosal invasion $(\mathrm{P}=0.666)$, skeinoid fibers $(\mathrm{P}=0.634)$, or higher risk category (high and intermediate risk category) $(\mathrm{P}=0.872)$ (Table III).

\section{Discussion}

The present study revealed unique laterally spreading features in $13 \%$ of a total of 52 GISTs. These spreading lesions were harbored within the otherwise normal-looking adjacent muscularis propria, so that recognition would be challenging either radiologically, endoscopically, or surgically. In fact, the present study did not reveal endoscopic features or signs specific to the laterally spreading lesions. These spreading lesions were not extensive (range, 0.12-0.7 cm), but infrequently $(29 \%$ of the 7 cases) involved the muscular surgical margins. These findings not only support the generally accepted concept that $\mathrm{R} 0$ resection requires a 1 to $2-\mathrm{cm}$ safety margin (8-10), but also indicate that such safety margins should be applied to the muscularis propria, not the mucosa or submucosa. In addition, the present study demonstrated a close relationship between laterally spreading lesions and the pedunculated shape of GISTs. Therefore, surgeons should pay attention to the $\geq 1$-cm muscular safety margins for R0 resection, particularly for pedunculated GISTs, even if these muscular walls are not thickened or tumorous.
In some cases, laterally spreading tumor cells were somewhat different from those of the main tumors. Compared with the pathological features of the main GISTs, laterally spreading lesions consisted of more slender tumor cells with smaller nuclei (in 3 cases), and showed weaker or diminished KIT/DOG $1^{+}$staining (in 3 cases). However, the former 3 cases were not identical to the latter 3 cases, and the slender morphological features of laterally spreading tumor cells did not appear to be associated with the weaker or diminished $\mathrm{KIT} / \mathrm{DOG}^{+}{ }^{+}$staining. On the other hand, the more slender morphological features of laterally spreading tumor cells somewhat resembled those of ICC hyperplasia $(4,6,13)$. It is known that ICCs can be positive for DOG1 $(14,15)$. These findings raise the possibility that some laterally spreading lesions may represent a hyperplastic reaction of ICCs caused, secondarily, by torque from the stalk of the pedunculated GISTs. However, the other 4 spreading lesions identified were composed of tumor cells resembling those of the main GISTs, suggesting true intramuscular extension of the tumor cells.

The most important risk factors associated with GISTs are anatomic location, tumor size, and mitotic activity, all of which are included in the widely accepted risk-stratification criteria $(1-6,12)$. Tumor rupture is another independent risk factor of GISTs, despite its rarity $(3-7,12)$. Mucosal invasion, high cellularity, and increased microvessel density may be additional indicators of poor prognosis $(1,3,4,6)$. Some authors noted high recurrence or poor prognosis in incomplete resection cases of GISTs compared with complete resection cases $(8,16)$. However, DeMatteo et al $(17)$ concluded that microscopically positive surgical margins did not influence patient outcomes. Zhi et al (18) demonstrated that a microscopically positive surgical margin can impact the disease-free 
survival of patients with GISTs, but had no influence on overall survival. Therefore, the prognostic significance of incomplete GIST resection remains controversial $(6,9,10,19)$. In the present study, both patients that had laterally spreading-related $\mathrm{R} 1$ resection were alive without recurrence $1-2.3$ years after surgery, demonstrating that they were not associated with unfavorable outcomes. However, the recurrence or metastases of GISTs may occur many years later (6), so that further follow-up is required. In addition, one patient with a laterally spreading GIST succumbed to the disease 2.5 years after surgery, although the surgical margin in this case was free of tumor cells and the spreading lesions exhibited ICC hyperplasia-like slender morphology. Therefore, regarding the clinicopathological significance of the laterally spreading lesions, further investigations of a larger series of GISTs are needed.

To the best of our knowledge, the present study is the first to histologically describe the laterally spreading features of GISTs, which occurred in $13 \%$ of the GISTs evaluated. These spreading lesions may contribute to $\mathrm{R} 1$ resection, albeit uncommonly. For R0 resection, the $\geq 1-\mathrm{cm}$ muscular safety margin should be required, particularly in cases of pedunculated GISTs.

\section{Acknowledgements}

The authors thank Mr. Kenji Okada and Mr. Shin-ichi Katori for their excellent technical assistance.

\section{Funding}

No funding was received.

\section{Availability of data and materials}

The datasets used during the present study are available from the corresponding author upon reasonable request.

\section{Authors' contributions}

SM conceived and designed the study. MM provided examined materials. SM, KM, and HT performed the histopathological examination. SM and YU collected appropriate references. SM wrote the paper. SM, MM, YU, KS, KM and HT reviewed and edited the manuscript. All authors read and approved the manuscript and agree to be accountable for all aspects of the research in ensuring that the accuracy or integrity of any part of the work are appropriately investigated and resolved.

\section{Ethics approval and consent to participate}

All experimental protocols were approved by the Medical Research Ethics Committees of the Japan Self-Defense Forces Central Hospital (Tokyo, Japan) and Mishuku Hospital (Tokyo, Japan).

\section{Consent for publication}

Not applicable.

\section{Competing interests}

The authors declare that they have no competing interests.

\section{References}

1. Miettinen M and Lasota J: Gastrointestinal stromal tumors: Pathology and prognosis at different sites. Semin Diag Pathol 23: 70-83, 2006.

2. Miettinen M, Fletcher CD, Kindblom LG and Tsui WM: Mesenchymal tumours of the oesophagus; mesenchymal tumours of the stomach; mesenchymal tumours of the small intestine; mesenchymal tumours of the colon and rectum. In: WHO Classification of Tumours of The Digestive System. Bosman FT, Carneiro F, Hruban RH and Theise ND (eds). 4th edition. International Agency for Research on Cancer, Lyon, pp35-36, pp74-79, pp115-116, pp181-182, 2010.

3. Rosai J (ed): Rosai and Ackerman's surgical pathology. 10th edition. Mosby/Elsevier, Philadelphia, PA, 2011.

4. Rubin BP: GIST and EGIST. In: Enzinger and Weiss's soft tissue tumors. Goldblum JR, Folpe AL and Weiss SW (eds). 6th edition. Elsevier/Saunders, Philadelphia, PA, pp569-590, 2014.

5. Miettinen MM, Corless CL, Debiec-Rychter M, Fletcher JA, Lasota J, Rubin BP and Sciot R: Gastrointestinal stromal tumor. In: WHO Classification of Tumours of Soft Tissue and Bone. Fletcher CDM, Bridge JA, Hogendoorn PCW and Mertens F (eds). 4th edition. International Agency for Research on Cancer, Lyon, pp164-167, 2013.

6. Grant NG and Noffsinger AE: Mesenchymal tumors. In: Fenoglio-Preiser's Gastrointestinal Pathology. Noffsinger AE (ed). 4th edition. Wolters Kluwer, Philadelphia, PA, pp1141-1222, 2017.

7. Dematteo RP, Heinrich MC, El-Rifai WM and Demetri G: Clinical management of gastrointestinal stromal tumors: Before and after STI-571. Hum Pathol 33: 466-477, 2002.

8. Gouveia AM, Pimenta AP, Capelinha AF, de la Cruz D, Silva P and Lopes JM: Surgical margin status and prognosis of gastrointestinal stromal tumor. World J Surg 32: 2375-2382, 2008.

9. Novitsky YW, Kercher KW, Sing RF and Heniford BT: Long-term outcomes of laparoscopic resection of gastric gastrointestinal stromal tumors. Ann Surg 243: 738-747, 2006.

10. Everett $M$ and Gutman H: Surgical management of gastrointestinal stromal tumors: Analysis of outcome with respect to surgical margins and technique. J Surg Oncol 98: 588-593, 2008.

11. Brierley JD, Gospodarowicz MK and Wittekind C: TNM classification of malignant tumours. 8th edition. Wiley-Blackwell, Hoboken, 2017.

12. Joensuu H: Risk stratification of patients diagnosed with gastrointestinal stromal tumor. Hum Pathol 39: 1411-1419, 2008.

13. Agaimy A and Wünsch PH: Sporadic Cajal cell hyperplasia is common in resection specimens for distal oesophageal carcinoma. A retrospective review of 77 consecutive surgical resection specimens. Virchows Arch 448: 288-294, 2006.

14. Espinosa I, Lee CH, Kim MK, Rouse BT, Subramanian S, Montgomery K, Varma S, Corless CL, Heinrich MC, Smith KS, et al: A novel monoclonal antibody against DOG1 is a sensitive and specific marker for gastrointestinal stromal tumors. Am J Surg Pathol 32: 210-218, 2008.

15. Miettinen M, Wang ZF and Lasota J: DOG1 antibody in the differential diagnosis of gastrointestinal stromal tumors: A study of 1840 cases. Am J Surg Pathol 33: 1401-1408, 2009.

16. Langer C, Gunawan B, Schüler P, Huber W, Füzesi L and Becker H: Prognostic factors influencing surgical management and outcome of gastrointestinal stromal tumours. Br J Surg 90: 332-339, 2003.

17. DeMatteo RP, Lewis JJ, Leung D, Mudan SS, Woodruff JM and Brennan MF: Two hundred gastrointestinal stromal tumors. Recurrence patterns and prognostic factors for survival. Ann Surg 231: 51-58, 2000.

18. Zhi X, Jiang B, Yu J, Røe OD, Qin J, Ni Q, Sun L, Xu M, Zhu J and Ma L: Prognostic role of microscopically positive margins for primary gastrointestinal stromal tumors: A systematic review and meta-analysis. Sci Rep 6: 21541, 2016.

19. McCarter MD, Antonescu CR, Ballman KV, Maki RG, Pisters PWT, Demetri GD, BlankeCD, von Mehren M,Brennan MF, McCall L, et al: Microscopically positive margins for primary gastrointestinal stromal tumors: Analysis of risk factors and tumor recurrence. J Am Coll Surg 215: 53-60, 2012. 